\title{
The Relationship Between Neurocognitive Function and Biomechanics: A Critically Appraised Topic
}

\author{
Ke'La Porter, Carolina Quintana, and Matthew Hoch
}

\begin{abstract}
Clinical Scenario: Neurocognitive performance may put individuals at a greater risk for lower-extremity musculoskeletal injuries. Research has observed the relationship between lower-extremity musculoskeletal injury and baseline neurocognitive performance; however, the understanding of this relationship is lacking. Exploring this relationship may give further insight into musculoskeletal injury and provide innovative directions for musculoskeletal injury prevention. Clinical Question: Is there a relationship between neurocognitive performance and lower-extremity biomechanics during a jumping or cutting task in healthy adult athletes? Summary of Key Findings: The literature was searched for articles that examined the relationship of a baseline neurocognitive test and a biomechanical analysis following a sports-related task. A total of 3 cross-sectional articles were included. All 3 studies concluded that poorer neurocognitive performance was associated with biomechanical faults that are linked to increased risk or rate of lower-extremity musculoskeletal injury. Clinical Bottom Line: Based on the evidence included, there is a moderate-level evidence to support the relationship between neurocognition and lower-extremity biomechanics in healthy adult athletes. Strength of Recommendation: In accordance with the van Tulder approach, there is a moderate level of evidence due to consistent findings from a combination of high- and limited-quality articles.
\end{abstract}

Keywords: head, brain, balance, posture

\section{Clinical Scenario}

Poor neurocognitive performance may be a risk factor for lower-extremity musculoskeletal injury risk. Previous research has identified poorer baseline neurocognitive function in college athletes who sustained anterior cruciate ligament injuries during an athletic season. ${ }^{1}$ Despite these findings, limited research exists attempting to understand the relationship between neurocognitive performance and musculoskeletal injuries. Neurocognitive performance contributes to several aspects of sport performance such as agility, fine motor skills, processing speed and reaction time, and dual tasking. ${ }^{2}$ Successfully integrating cognitive demands during sport participation is critical for rapid decision making, reacting to opponents, and ball handling. ${ }^{2,3}$ A decreased ability to rapidly process cognitive information such as spatial orientation or visual stimuli and make rapid decisions may increase coordination errors and detract from the ability to evade injury..$^{2,3}$ Recent research has assessed the relationship between baseline neurocognitive performance and lower-extremity biomechanics, such as ground reaction forces and knee and hip kinematics during athletic tasks. Understanding the relationship between neurocognition and lower-extremity biomechanics may provide insight into musculoskeletal injury and innovative directions for injury prevention.

The authors are with the Department of Athletic Training and Clinical Nutrition, College of Heath Sciences, Sports Medicine Research Institute, University of Kentucky, Lexington, KY, USA. Hoch (Matt.Hoch@uky.edu) is corresponding author.

\section{Focused Clinical Question}

Is there a relationship between neurocognitive performance and lower-extremity biomechanics during a jumping or cutting task in healthy adult athletes?

\section{Search Strategy}

The computerized search was conducted in September 2019 (Figure 1). The following search terms were used:

- athlete OR sport

- cogniti*OR neurocogni* OR memory OR reaction time

- biomechanics OR mechanics OR cutting OR landing

- lower-extremity OR lower limb

\section{Sources of Evidence Searched}

- EBSCOhost

- MEDLINE

- SPORTDiscus

- Health source-consumer edition

- Additional resources obtained via hand search of reference lists

\section{Inclusion Criteria}

- Participants completed at least one neurocognitive test

- Participants completed a sports-related task (ie, jumping/ landing, cutting) with lower-extremity biomechanical analysis (ie, kinematics, kinetics) 


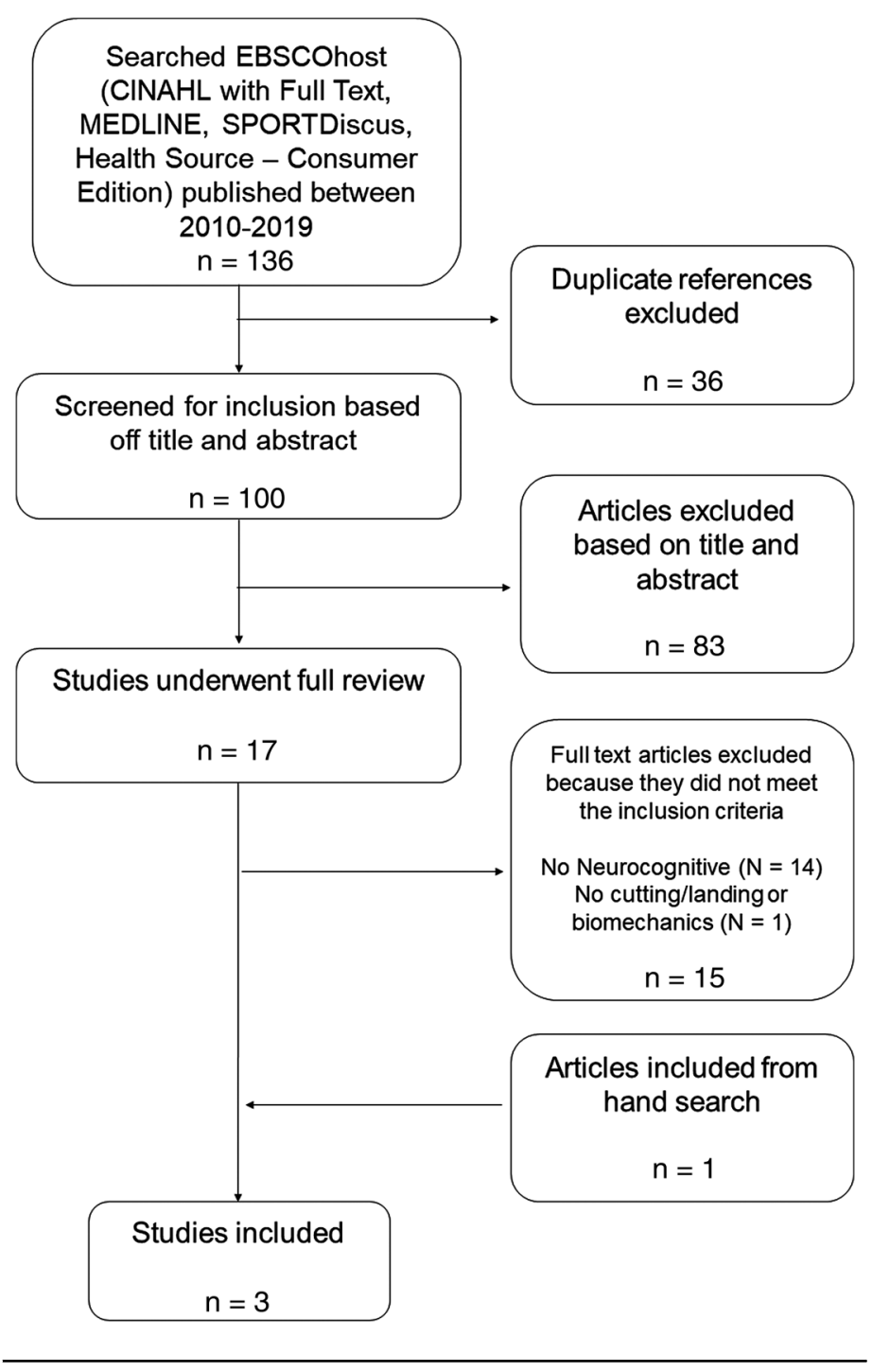

Figure 1 - Flow diagram of systematic search of the literature.

- Participants were recreational or competitive athletes with no current injuries

- Studies published in English

- Studies that were level 3 evidence based on Oxford Center of Evidence-Based Medicine

- Studies published within the last 10 years.

\section{Exclusion Criteria}

- Studies that examined dual tasks by adding cognitive load to sport-related tasks

- Studies that did not utilize a neurocognitive test

- Reviews of the literature, expert opinions, case studies/ series.

\section{Evidence Quality Assessment}

The Downs and Black Reduced Checklist ${ }^{4,5}$ was used to critically appraise the included studies. There were 16 components to the checklist. Scores of $\geq 75 \%(12 / 16)$ were considered high-quality studies. Two authors (K.P. and C.Q.) independently reviewed and scored the included studies. Following independent review, the 2 authors (K.P. and C.Q.) reviewed the completed appraisals and came to a consensus regarding the quality of the studies.

\section{Results of Search}

\section{Summary of Search, Best Evidence Appraised, and Key Findings}

- The literature was searched for studies that correlated performance on neurocognitive tests to lower-extremity biomechanics during a jumping or cutting task.

- The search returned 100 results. After review of the titles and abstracts, 83 articles were excluded, and the remaining 17 articles underwent review of the full text. Two articles met the inclusion/exclusion criteria, and 1 article was added from a hand search for a total of 3 articles to be included in this critically appraised topic (CAT).

- Two of the studies ${ }^{2,6}$ compared the lower-extremity biomechanics in high and low neurocognitive performance groups. The remaining study ${ }^{7}$ explored the relationship between neurocognitive performance and knee biomechanics when ball handling and nonball handling.

- All 3 studies ${ }^{2,6,7}$ concluded that poorer neurocognitive performance was associated with biomechanics linked to elevated lower-extremity musculoskeletal injury risk following a jumplanding/cutting task, such as increased knee valgus and ground reaction force.

\section{Results of Evidence Quality Assessment}

The Downs and Black Reduced Checklist ${ }^{4,5}$ was used to critically appraise the included studies. Monfort et $\mathrm{al}^{7}$ received a 13/16, Herman and Barth ${ }^{2}$ received a $11 / 16$, and Shibata et $\mathrm{al}^{6}$ received a 13/16 (Table 1). Among the limitations of the quality assessments were the lack of blinding of those measuring the main outcomes in each of the included studies. Another limitation was the lack of described confounders. While Herman and Barth ${ }^{2}$ and Monfort et $\mathrm{al}^{7}$ clearly describe the method of their outcome measures in detail, they failed to include or report the accuracy, reliability, and validity of the measures. Herman and Barth ${ }^{2}$ did not report actual probability values for all main outcomes. Finally, a point was deducted from Shibata et $\mathrm{al}^{6}$ for lack of adjustments for the potential confounders in the analysis from which the main findings were drawn.

\section{Clinical Bottom Line}

An adaptation of the van Tulder approach was used to determine the strength of recommendation for the clinical question. According to the van Tulder approach, ${ }^{8}$ the strength of recommendation can be strong, moderate, limited, or conflicting based on the consistency and quality of evidence. Based on the 3 articles included (Table 2), there was a moderate-level evidence to support the relationship between neurocognition and lower-extremity biomechanics in healthy adult athletes. This recommendation was selected because there were consistent findings from a combination of high- and limited-quality articles. 
Table 1 Summary of Evidence

\begin{tabular}{llcl}
\hline $\begin{array}{l}\text { Level of } \\
\text { evidence }\end{array}$ & $\begin{array}{l}\text { Study } \\
\text { design }\end{array}$ & $\begin{array}{l}\text { Number } \\
\text { located }\end{array}$ & Author \\
\hline $3 \mathrm{~B}$ & $\begin{array}{l}\text { Cross- } \\
\text { sectional }\end{array}$ & 3 & Monfort et al \\
& & & $\begin{array}{l}\text { Herman and } \\
\text { Barth }^{2} \\
\end{array}$ \\
& & Shibata et $\mathrm{al}^{6}$ \\
\hline
\end{tabular}

\section{Implications for Practice, Education, and Future Research}

Evidence has demonstrated that there is a relationship between neurocognitive performance and biomechanical function following a jumping or cutting task. This relationship was identified despite the variability in the biomechanical assessments, neurocognitive testing, and patient population across the included studies. Despite moderate evidence and low sample sizes reported, these findings may have implications for clinical practice because baseline neurocognitive testing is currently utilized in many athletic settings and may provide novel insights into musculoskeletal injury prevention and rehabilitation.

All 3 studies identified increased peak knee valgus angle and moment, and increased ground reaction forces in individuals with poorer neurocognitive performance. Monfort et $\mathrm{al}^{7}$ studied knee valgus angle, knee valgus moment, and ground reaction force during a cutting motion with ball handling and nonball handling. Herman and Barth ${ }^{2}$ and Shibata et $\mathrm{al}^{6}$ both started the participants on a $30-\mathrm{cm}$ box, jumping to the ground with both feet, followed by an unanticipated $45^{\circ}$ cutting or vertical landing task. For the variables used to investigate the relationships, Herman and $\mathrm{Barth}^{2}$ reported ground reaction force, knee abduction moment, and knee adduction-abduction joint angles. Shibata et $\mathrm{al}^{6}$ reported knee valgus angle, knee valgus moment, and ground reaction force.

Despite variance in the tasks, all 3 studies identified alterations in biomechanics associated with poorer neurocognitive performance. Monfort et $\mathrm{al}^{7}$ reported confidence intervals in the comparison of ball handling versus nonball handling and peak knee valgus angle. As the $95 \%$ confidence interval did not encompass 0 , this provided additional confidence in the results. In addition, Herman and Barth ${ }^{2}$ reported differences between the high- and low-performing groups and noted significant group differences. However, it should be noted that knee valgus, knee moment, knee flexion, hip adduction, and ground reaction forces did not reach a level of statistical significant in the investigation by Shibata et al. ${ }^{6}$ To further examine the results of Shibata et al, ${ }^{6}$ effect sizes were utilized to further examine the trends in the data. The effect sizes associated with most of these outcomes in the Shibata et $\mathrm{al}^{6}$ study were moderate to large indicating clinically relevant differences may be present between the high and low cognitive performance groups. This can be attributed to the small sample size limiting the ability to detect statistically significant differences. Despite the variability in the biomechanical assessments across studies, the authors can conclude that biomechanical patterns exhibited by poor cognitive performers were not a result of the testing methods.

In addition to utilizing different biomechanical tasks, the relationship between neurocognition and biomechanics remained true despite the included studies utilizing different methods to test neurocognition. Monfort et $\mathrm{al}^{7}$ utilized a computerized test, the Immediate Post-Concussion Assessment and Cognitive Testing, for the neurocognitive test. The Immediate Post-Concussion Assessment and Cognitive Testing provides a composite score for 4 different domains including visual memory, verbal memory, reaction time, and processing speed, ${ }^{9}$ and despite its popularity and common clinical uses, the test has been reported to have low to moderate test-retest reliability in a healthy sample (intraclass correlation coefficient $=$ .39-.61). ${ }^{10}$ Herman and Barth ${ }^{2}$ utilized a computerized neurocognitive test, known as the concussion resolution index which included 6 domains that were broken down into the following 3 groups: simple reaction time, complex reaction time, and processing speed. In previous investigations, the concussion resolution index has demonstrated low to moderate test-retest reliability in healthy individuals (intraclass correlation coefficient $=.03-.66$ ). ${ }^{10}$ Shibata et al ${ }^{6}$ utilized a pen and paper neurocognitive test known as the Symbol Digit Modalities Test. The Symbol Digit Modalities Test examined psychomotor speed, visual short-term memory, attention, and concentration. ${ }^{11}$ To the authors' knowledge, the reliability and validity have not been reported in a healthy, athletic population.

Following testing, Herman et $\mathrm{al}^{2}$ and Shibata et $\mathrm{al}^{6}$ split their participants into high- or low-performing groups. Herman and Barth $^{2}$ used the results of multiple constructs to form a group of high and low performers. Shibata et $\mathrm{al}^{6}$ took a more basic approach, looking at the average achievement rate, then using the median as a cutoff between groups. While these 3 tests all have different components, price range, and time demands, they all effectively challenged neurocognitive function. This indicates that various baseline neurocognitive testing may be able to provide insight into lower-extremity biomechanical patterns that may predispose individuals to injury.

The patient population was also diverse across the 3 studies. Monfort et $\mathrm{al}^{7}$ included all males, Shibata et $\mathrm{al}^{6}$ included all females, and Herman and Barth ${ }^{2}$ included both male and female participants. It did not appear that gender played a role in the outcome of the studies. In addition, the type of athletic participation varied in the participants included across all of the studies. Monfort et $\mathrm{al}^{7}$ recruited athletes from the men's college club soccer team, Herman and Barth $^{2}$ and Shibata et $\mathrm{al}^{6}$ included nonspecific athletes who participated in jumping/cutting sports with recreational and university club athletics, respectively. While the level of participation was similar, the sports they participated in represented a more diverse sample. Despite these differences in sports participation, all 3 studies came to the same conclusion regarding the relationship between neurocognition and biomechanics. This suggests that the findings of the CAT may have application to a wide array of athletic settings, however, limited to college-aged athletes.

Currently, it is common practice to administer baseline concussion testing for athletes before beginning sports participation. Using these baseline scores, it may be possible to identify individuals with poor neurocognitive test results for further assessment of biomechanics for the purposes of musculoskeletal injury prevention. However, there is a wide range of tools used to measure neurocognitive performance. While many generate a percentile score that could quantify poor, average, or good neurocognitive test results, this may not be the case for all testing batteries. Future research should determine the best way to systematically define poor performers, whether this entails the use of a single-standardized test or defining cutoff scores for multiple tests. 
Table 2 Characteristics of Studies Selected

\begin{tabular}{ll}
\hline & ${\text { Monfort } \text { et }^{\text {al }}}^{\mathbf{7}}$ \\
\hline Study design & Cross-sectional \\
Study title & Visual-spatial memory deficits are related \\
& to increased knee valgus angle during a \\
& sport-specific sidestep cut
\end{tabular}

Participants

15 male athletes (mean age 20.7 [2.0] y, height 1.78 [0.07] $\mathrm{m}$; weight 76.5 [8.9] $\mathrm{kg}$ )

Inclusion/exclusion criteria

Inclusion: current members of the male college club indoor soccer team or had been members in the past $2 \mathrm{mo}$; no history of traumatic (resulting in surgery and time loss) knee/ankle injury or minor injuries (hindering but not resulting in time loss) in the past 3 mo that limited their participation; Tegner Activity Scale $\geq 7$ and Marx Activity Scale $\geq 12$

Exclusion: no specific exclusion criteria were noted

Methods

Outcome

measures

Herman and Barth $^{2}$
Cross-sectional
Drop-jump landing varies with baseline
neurocognition: implications from ACL
injury risk and prevention

\section{7 athletes (age 18-30)}

High-performance group: mean age 21.05 (1.54) y; height $1.73(0.07) \mathrm{m}$; weight $68.68(9.26) \mathrm{kg}$

Low-performance group: mean age 20.81 (1.68) y; height $1.71(0.12) \mathrm{m}$; weight $70.11(10.12) \mathrm{kg}$

Inclusion: recreational athletes who participate in jumping/cutting sports at least 3 times a week OR participate in these sports at least once a month and previously participated in high school varsity or college club levels

Exclusion: did not meet age criteria, did not meet the activity-level criteria, had a lower-extremity injury that prevented participation for longer than 2 wk over the previous 6 mo, possessed cardiovascular, respiratory, neurological, or other conditions that prevented them from partici-

pating at maximal effort, had suffered a concussion within the last year

Participants completed the CRI that included 6 subset domains: the reaction time, the cued reaction time, the animal decoding, the visual recognition 1 and 2 , and the symbol scanning subsets. They were then separated into HP and LP. Participants stood on 30-cm box placed at a distance measuring one-half of the subject's height away. The subject stands on the box, jumps forward off the box with both feet, lands on the target, then jumps at maximum effort to the second target. The second target was revealed after leaving the $30-\mathrm{cm}$ box, pointing 1 of 3 directions (left $45^{\circ}$ angle, vertical, right $45^{\circ}$ angle). 16 retroreflective markers were placed over the trunk, pelvis, and lowerextremity. The marker data was collected using a motion analysis system. Ground reaction forces were obtained using force plates, in conjunction with the motion capture data.

Biomechanical outcomes

- Ground reaction force

- Knee adduction-abduction moment

- Knee flexion and adduction-abduction joint angles

- Hip flexion and adduction-abduction

joint angles

- Trunk flexion and lateral bending angles
Shibata et $\mathbf{a l}^{6}$

Cross-sectional

The influence of differences in neurocognitive function on lower limb kinematics, kinetics, and muscle activity during an unanticipated cutting motion

15 female athletes (mean age 20.1 [1.3] $\mathrm{y}$; height 1.67 [0.7] m; weight 60.6 [6.9] kg)

Inclusion: play sports including jumping/ cutting motions; practicing $2-3 \mathrm{~h}$ a day, 5-6 d a week

Exclusion: injuries in the lower limb, a concussion within the past $6 \mathrm{mo}$, any disorder of the peripheral sensory system, past history of surgery in the lumbar spine or lower limbs

Participants were administrated the SDMT and grouped into a HP or LP group based on their achievement rate.

Participants jumped off a $30-\mathrm{cm}$ high box, then performed a random task according to a command on a personal computer monitor immediately after leaving the box. The tasks included a side-step cutting 45 , single-leg landing, and forward stepping using the dominant limb. 35 retroreflective markers were placed over the whole body. A motion analysis system was used to capture the motions. Ground reaction forces were obtained using a force platform that was synchronized with the kinematic data. Surface EMG was recorded using a 7-channel EMG system and collected synchronously with motion and force plate data.

Biomechanical outcomes

- Ground reaction force

- Surface EMG

- Hip flexion, adduction, internal rotation angle

- Knee flexion and valgus angle

- Hip flexion and adduction moment

- Knee flexion and abduction moment 
Table 2 (continued)

\begin{tabular}{ll}
\hline Monfort et al \\
\hline Results & Worse visual-spatial memory was asso- \\
ciated with a greater pKVA $\left(R^{2}=52 \%\right.$, & adjusted $\left.R^{2}-48 \%, P=.008\right)$. Visual \\
& memory composite score was the stron- \\
& gest predictor for change in pKVM but did \\
& not reach statistical significance after \\
& controlling for multiple comparisons \\
& $\left(R^{2}=27 \%\right.$, adjusted $\left.R^{2}=21 \%, P=.049\right)$. \\
& BH had a significant group effect on \\
& pKVA $(P=.02)$, having a decrease in \\
& pKVA (mean: $-1.6^{\circ} ; 95 \%$ CI, -3 to $-0.3 ;$ \\
& Cohen $d: 0.67)$ compared with NB. No \\
& significant effect was observed for \\
& pKVM.
\end{tabular}

Conclusion

Neurocognitive test scores on visualspatial memory were associated with neuromuscular control during a dripping task, more specifically increased knee valgus. Assessing this neurocognitive domain may be useful in identifying athletes with reduced neuromuscular control.

Level of $\quad 3 B$

evidence

Validity scores $13 / 16$

Support for the Yes

\section{Herman and Barth ${ }^{2}$}

The LP group demonstrated significantly lower CRI scores in all domains compared with the HP group. Processing speed was a $20.3 \%$ difference, simple reaction time was $42.6 \%$ difference, and complex reaction time was $47.1 \%$ difference. The LP group had a $31 \%$ higher peak vertical ground reaction force and $26 \%$ higher peak proximal anterior tibial shear force. The LP group also had a difference of $4.8^{\circ}$ in knee abduction angle.

Athletes who performed poorer on baseline neurocognitive assessments also demonstrated knee kinetics and kinematics that are related to ACL injury. Assessing neurocognitive performance may aid in the identification of those are elevated injury risk.

3B

$12 / 16$

yes

\section{Shibata et al $^{6}$}

The average SDMT achievement rate was $65.0 \%(6.8 \%)$. The top 7 were placed in the HP group (achievement rate $70.8 \%$ [5\%]), the remaining 8 were placed in the LP group $(60.0 \%$ [3.1\%]). The knee extensors (QUAD) activity was larger in the LP group compared with the HP group at $50 \mathrm{~ms}$ before initial contact (pre-IC) and the first $50 \mathrm{~ms}$ of the cutting phase (postIC). The CCR was larger at pre-IC and significantly larger at post-IC. When looking at the

$P$ value for peak kinematics and kinetics, it showed no statistical significance. However, the effect size for knee valgus was 0.49 and the effect size for knee moment, knee flexion, and hip flexion were $0.77,0.90$, and 0.96 , respectively.

Lower performance on the SDMT was associated with quadriceps dominant muscle activity that has been reported to increase the load on the ACL, presenting a potential increased risk.

3B

$13 / 16$

yes

Abbreviations: ACL, anterior cruciate ligament; BH, ball handling; CCR, co-contraction ratio; CRI, concussion resolution index; EMG, electromyography; HP, high performance; ImPACT, Immediate Post-Concussion Assessment and Cognitive Testing; LP, low performance; NB, nonball ; pKVA, peak knee valgus angle; pKVM, peak knee valgus moment; SDMT, Symbol Digit Modalities Test.

Future research should examine changes in biomechanics following neurocognitive training. As previously mentioned, there are different ways to effectively measure neurocognitive performance; therefore, there are also different means of challenging neurocognition. This preventative approach could challenge aspects such as reaction time, visual memory, and processing speed. Wilkerson et al ${ }^{12}$ assessed and trained visuomotor reaction time in college football players. Assessment and training were completed using a visuomotor reaction time system (VMRT) with 64 target buttons to press when illuminated, tracking the average number of target hits, and the amount of elapsed time between hits. ${ }^{12}$ First, after administering preseason baseline testing then tracking injuries over the course of the season, $52 \%$ of the slower players sustained a strain or sprain versus $32 \%$ of the faster players. ${ }^{12}$ In addition, after implementing a visuomotor training program during the postseason, there was an improvement in reaction time performance in the slow performers group but not in the fast performers group. ${ }^{12}$ This may suggest that VMRT may be a modifiable risk factor to injury, but there may be a cap on the maximum level of performance improvements. These results identify an area for further investigation to identify how this translates to injury risk, prevention, and rates as it is an intervention that can be widely applied. Similar methods could be employed to track the implementation of a neurocognitive training. As previously mentioned, neurocognition takes many forms. This training program could employ similar reaction time methods as Wilkerson et al, ${ }^{12}$ it could come in the form of memory training games, or it could challenge processing speed. To take it a step further, if the primary neurocognitive test could identify which portion of neurocognition players are weakest in, the training could be more focused. However, these interpretations must be made with caution as it is unknown if biomechanical faults improve or if injury rates improve following training.

Based on the literature reviewed in this CAT, a relationship between neurocognition and lower-extremity biomechanics has been repeatedly demonstrated. With different methods utilized for biomechanical assessment, neurocognitive evaluation, and patient recruitment, all 3 articles included in this CAT yielded similar results. Education about neurocognitive testing and standardizing the results needs to be further developed before intervention strategies can be implements. Future research should look at the effects of neurocognitive intervention on biomechanics.

\section{Acknowledgments}

No financial support was used for this investigation. The authors declare no conflict of interest.

\section{References}

1. Swanik C, Covassin T, Stearne D, Schatz P. The relationship between neurocognitive function and noncontact anterior cruciate ligament 
injuries. Am J Sports Med. 2007;35(6):943-948. PubMed ID: 17369562 doi:10.1177/0363546507299532

2. Herman DC, Barth JT. Drop-jump landing varies with baseline neurocognition: implications for anterior cruciate ligament injury risk and prevention. Am J Sports Med. 2016;44(9):2347-2353. PubMed ID: 27474381 doi:10.1177/0363546516657338

3. Herman DC, Zaremski JL, Vincent HK, Vincent KR. Effect of neurocognition and concussion on musculoskeletal injury risk. Curr Sports Med Rep. 2015;14(3):194-199. doi:10.1249/JSR. 0000000000000157

4. Munn J, Sullivan SJ, Schneiders AG. Evidence of sensorimotor deficits in functional ankle instability: a systematic review with meta-analysis. J Sci Med Sport. 2010;13(1):2-12. PubMed ID: 19442581 doi:10.1016/j.jsams.2009.03.004

5. Downs SH, Black N. The feasibility of creating a checklist for the assessment of the methodological quality both of randomised and non-randomised studies of health care interventions. J Epidemiol Community Health. 1998;52(6):377-384. PubMed ID: 9764259 doi:10.1136/jech.52.6.377

6. Shibata S, Takemura M, Miyakawa S. The influence of differences in neurocognitive function on lower limb kinematics, kinetics, and muscle activity during an unanticipated cutting motion. Phys Ther Res. 2018;21(2):44-52. PubMed ID: 30697509 doi:10.1298/ptr. E9938
7. Monfort SM, Pradarelli JJ, Grooms DR, Hutchison KA, Onate JA, Chaudhari AMW. Visual-spatial memory deficits are related to increased knee valgus angle during a sport-specific sidestep cut. Am J Sports Med. 2019;47(6):1488-1495. PubMed ID: 30986095 doi:10. $1177 / 0363546519834544$

8. van Tulder M, Furlan A, Bombardier C, Bouter L., \& Editorial Board of the Cochrane Collaboration Back Review Group. Updated method guidelines for systematic reviews in the cochrane collaboration back review group. Spine. 2003;28(12):1290-1299. PubMed ID: 12811274

9. Schatz P, Pardini JE, Lovell MR, Collins MW, Podell K. Sensitivity and specificity of the ImPACT Test Battery for concussion in athletes. Arch Clin Neuropsychol. 2006;21(1):91-99. PubMed ID: 16143492 doi:10.1016/j.acn.2005.08.001

10. Broglio SP, Ferrara MS, Macciocchi SN, Baumgartner TA, Elliott R. Test-retest reliability of computerized concussion assessment programs. J Athl Train. 2007;42(4):509. PubMed ID: 18174939

11. Register-Mihalik JK, Kontos DL, Guskiewicz KM, Mihalik JP, Conder $\mathrm{R}$, Shields EW. Age-related differences and reliability on computerized and paper-and-pencil neurocognitive assessment batteries. J Athl Train. 2012;47(3):297-305. doi:10.4085/1062-6050-47.3.13

12. Wilkerson GB, Simpson KA, Clark RA. Assessment and training of visuomotor reaction time for football injury prevention. J Sport Rehabil. 2017;26(1):26-34. PubMed ID: 27632871 doi:10.1123/jsr.2015-0068 